\title{
Anti-HMGCR Autoantibodies in Juvenile Idiopathic Inflammatory Myopathies Identify a Rare but Clinically Important Subset of Patients
}

\author{
Sarah L. Tansley, Zoe E. Betteridge, Stefania Simou, Thomas S. Jacques, Clarissa Pilkington, \\ Mark Wood, Kishore Warrier, Lucy R. Wedderburn, and Neil J. McHugh, on behalf of the \\ Juvenile Dermatomyositis Research Group
}

\begin{abstract}
Objective. We aimed to establish the prevalence and clinical associations of anti-HMG-CoA-reductase (anti-HMGCR) in a large UK cohort with juvenile myositis.

Methods. There were 381 patients investigated for anti-HMGCR using ELISA.

Results. Anti-HMGCR autoantibodies were detected in 4 patients (1\%). These children had no or minimal rash and significant muscle disease. Muscle biopsies were considered distinctive, with widespread variation in fiber size, necrotic fibers, and chronic inflammatory cell infiltrates; all had prolonged elevation of creatine kinase and all ultimately received biologic therapies.

Conclusion. Anti-HMGCR in UK children with myositis are associated with severe disease that is poorly responsive to standard treatment. (First Release February 15 2017; J Rheumatol 2017;44:488-92; doi:10.3899/jrheum.160871)
\end{abstract}

Key Indexing Terms: PEDIATRIC DERMATOMYOSITIS/POLYMYOSITIS AUTOANTIBODIES PEDIATRIC RHEUMATIC DISEASES
PROGNOSIS

\footnotetext{
From the Royal National Hospital for Rheumatic Diseases, Royal United Hospitals Foundation Trust; University of Bath, Bath; University College London (UCL) Institute of Child Health; Arthritis Research UK Centre for Adolescent Rheumatology at UCL, University College London Hospitals (UCLH), and Great Ormond Street Hospital (GOSH), London; Leeds Children's Hospital, Leeds; Nottingham University Hospitals UK National Health Service (NHS) Trust, Nottingham, UK.

Funding for the UK Juvenile Dermatomyositis Cohort and Biomarker study has been provided by the Wellcome Trust UK (085860), Action Medical Research UK (SP4252), The Myositis Support Group UK, Arthritis Research UK (14518, 20164), The Henry Smith Charity and Great Ormond Street Children's Charity (V1268), and the National Institute for Health Research (NIHR) Translational Research Collaboration Rare Diseases. This research was supported by the NIHR Biomedical Research Centre at GOSH for Children NHS Foundation Trust and Institute of Child Health UCL. SLT is supported by a fellowship from The Bath Institute for Rheumatic Diseases and has received previous support from the BMA Doris Hillier Grant (2012).

S.L. Tansley, MBChB, MRCP, Royal National Hospital for Rheumatic Diseases, Royal United Hospitals Foundation Trust, and University of Bath; Z.E. Betteridge, BSc, PhD, University of Bath; S. Simou, MSc, UCL Institute of Child Health; T.S. Jacques, PhD, MRCP, FRCPath, UCL Institute of Child Health, and GOSH; C. Pilkington, MBBS, FRCPCH, FRCP, GOSH; M. Wood, MBBS, MRCPCH, Leeds Children's Hospital; $K$. Warrier, MBBS, MRCPCH, Nottingham University Hospitals NHS Trust; L.R. Wedderburn, MD, PhD, FRCP, UCL Institute of Child Health, and GOSH, and Arthritis Research UK Centre for Adolescent Rheumatology at UCL, UCLH, and GOSH; N.J. McHugh, MD, FRCP, FRCPath, Royal National Hospital for Rheumatic Diseases, Royal United Hospitals Foundation Trust, and University of Bath.

Address correspondence to Dr. S.L. Tansley, Royal National Hospital For Rheumatic Diseases NHS Foundation Trust, Upper Borough Walls, Bath BA11RL,UK.E-mail: s.tansley@nhs.net

Full Release Article. For details see Reprints and Permissions at jrheum.org Accepted for publication December 23, 2016.
}

Necrotizing autoimmune myopathy (NAM) is a subgroup of the idiopathic inflammatory myopathies that is defined by common clinical and histopathological features. Patients present with high creatinine kinase (CK) levels and often profound weakness. On muscle biopsy, characteristic features include myofiber necrosis, minimal endomysial, and perivascular inflammatory infiltrate, and in some cases focal endomysial fibrosis ${ }^{1}$. Despite little or no muscle inflammation, NAM usually responds to immunomodulatory therapy, highlighting that this is an immune-mediated phenotype. It has been associated with 2 myositis-specific autoantibodies: antisignal recognition peptide (anti-SRP) and more recently anti-HMG-CoA-reductase (HMGCR).

Patients with anti-HMGCR-associated disease make up about $6 \%$ of adult idiopathic inflammatory myopathy cohorts $^{2}$. In addition to NAM, anti-HMGCR antibodies are associated with statins, an intriguing finding given that HMGCR is the pharmacologic target of statins and is upregulated by statin use ${ }^{2}$. While statins are typically prescribed to adult patients, anti-HMGCR has been reported in patients with juvenile-onset NAM, but data are extremely limited ${ }^{3}$. Further, $40 \%-70 \%$ of adults with anti-HMGCR-associated NAM have no history of statin exposure, suggesting the presence of alternative disease triggers ${ }^{2,3}$. We aimed to establish the prevalence and clinical associations of anti-HMGCR in a large UK juvenile idiopathic inflammatory myositis (JIIM) cohort.

Personal non-commercial use only. The Journal of Rheumatology Copyright @ 2017 . All rights reserved. 


\section{MATERIALS AND METHODS}

Patients. Serum samples and matched clinical data were obtained from 381 patients with JIIM recruited to the UK Juvenile Dermatomyositis Cohort and Biomarker Study described previously ${ }^{4}$. Ethical approval has been obtained (Northeast-York Research Ethics Committee 01/3/022). Parental consent and consent or age-appropriate assent was obtained in accordance with the Declaration of Helsinki.

Autoantibody detection. Immunoprecipitation of all samples was performed to determine the presence of myositis-specific and -associated autoantibodies, as previously described ${ }^{5,6}$. The presence of anti-HMGCR was determined in all samples by ELISA using recombinant antigen, as previously described $^{7}$. Positive samples were confirmed by Western blotting (insufficient serum for patient 3). A further 48 juvenile healthy controls, 21 patients with juvenile systemic lupus erythematosus, and 27 muscular dystrophy were found to be negative for anti-HMGCR.

Immunofluorescence. Human epithelial cell line 2 indirect immunofluorescence was performed at 1:40 serum dilution for all anti-HMGCR-positive samples according to the manufacturer's instructions (Inova).

Muscle biopsy. Where tissue was available for reassessment, muscle biopsies were processed, stained, and scored (using the International Juvenile Dermatomyositis score tool) by an expert pediatric neuropathologist (TSJ) blinded to clinical data ${ }^{8,9}$

\section{RESULTS}

Anti-HMGCR autoantibodies were detected in 4 of the 381 patients $(1 \%)$. None had a history of statin exposure. Anti-HMGCR autoantibodies were not found in conjunction with any other myositis-specific or -associated autoantibody. All patients with anti-HMGCR had a cytoplasmic staining pattern on immunofluorescence, and 2 had additional coarse speckled nuclear staining.

While numbers were insufficient for statistical analysis, based on Childhood Myositis Assessment Scores (CMAS) and the physician's global assessment of disease activity visual analog scores (PGA), children with anti-HMGCR were weaker and had greater disease activity (Table 1). Consistent with previous reports in juvenile-onset disease, CK at presentation was elevated in just $55.4 \%$ of patients ${ }^{10,11}$. Of those patients with an elevated CK, the median value was $1039 \mathrm{IU} / 1$
(415-4115.25), compared with $15,500(12,000-25,250)$ for those with anti-HMGCR.

Patient 1. A 4-year-old girl developed a relatively slow-onset muscular weakness with a lowest-ever CMAS of $28(0-53)$, corresponding to a moderate degree of weakness ${ }^{12}$. CK at presentation was 12,000 . She had no rash. Muscle biopsy was reported to be consistent with dermatomyositis (DM). Her highest-ever PGA was 4.8 out of 10. She responded to initial treatment with oral methotrexate (MTX) and steroids, but CK remained elevated. Her disease flared (lowest CMAS of 33 with no rash and peak CK of 7000) when MTX was stopped after a lengthy period of remission, following which treatment was restarted with subcutaneous MTX alongside oral prednisolone (PRED). She subsequently responded to infliximab (IFX) and entered remission.

Patient 2. A 13.8-year-old girl presented with periorbital puffiness and hyperpigmentation with progressive weakness over 1-2 months. Her CK was found to be 44,000 . She received pulsed intravenous (IV) methylprednisolone with some improvement. Muscle biopsy revealed a destructive myopathy with inflammation and necrosis. One month later, her CMAS score was 0 , indicating very severe weakness. She required nasogastric feeding. She received further pulses of IV methylprednisolone, cyclophosphamide (CYC), and subcutaneous MTX. Improvement in muscle power was extremely slow, leading to concern that there may be an additional underlying muscle disease. Further investigations, including magnetic resonance imaging (MRI), electromyography, and nerve conduction studies were consistent with myositis. This patient was subsequently treated with azathioprine, rituximab (RTX), and further CYC before entering remission with IFX.

Patient 3. An 11.8-year-old boy presented with a 6-month history of evolving proximal muscle weakness. CK was 19,000. Lack of any rash prompted consideration of muscular

Table 1. Summary of the characteristics of the 4 patients with anti-HMGCR autoantibodies compared with the remainder of the JIIM cohort. Patients with anti-HMGCR were weaker, had more severe disease, and had dramatically higher CK levels throughout their disease course.

\begin{tabular}{|c|c|c|c|c|c|c|c|c|c|}
\hline Patient & $\begin{array}{l}\text { Skin } \\
\text { Rash }\end{array}$ & Sex & $\begin{array}{c}\text { Age at } \\
\text { Disease Onset, } \\
\text { Yrs }\end{array}$ & $\begin{array}{c}\text { Lowest CMAS, } \\
0-53\end{array}$ & $\begin{array}{c}\text { Highest PGA, } \\
0-10\end{array}$ & $\begin{array}{c}\text { CK at Onset, } \\
\text { IU/1 }\end{array}$ & $\begin{array}{c}\text { CK at } 6 \text { Mos } \\
\text { Postdiagnosis, } \\
\text { IU/1 }\end{array}$ & $\begin{array}{c}\mathrm{CK} \text { at } 12 \mathrm{Mos} \\
\text { Postdiagnosis, } \\
\text { IU/1 }\end{array}$ & $\begin{array}{l}\text { Lowest CK, } \\
\text { IU/l }\end{array}$ \\
\hline 2 & Minimal & Female & 13 & 0 & 8.3 & 44,002 & 404 & 13,065 & 251 \\
\hline 3 & No & Male & 11 & 36 & 3 & 19,000 & 4702 & 4038 & 1527 \\
\hline 4 & Minimal & Female & 9 & 2 & 9.5 & 12,662 & 2004 & 4638 & 2241 \\
\hline \multicolumn{3}{|c|}{$\begin{array}{l}\text { Median (IQR) for all } \\
381 \text { patients }\end{array}$} & $6.8(3.9-10.1)$ & $40(24-47.3)$ & $4(2.2-7)$ & $225(78-1191.5)^{\mathrm{a}}$ & $65(41-106)^{\mathrm{b}}$ & $88(60-114)^{\mathrm{c}}$ & $53(37-82.5)$ \\
\hline
\end{tabular}

${ }^{a}$ Median for those with a raised CK 1039 (415.4-115.25). ${ }^{\mathrm{b}} \mathrm{CK}$ available between 4 and 8 months for 60 patients. ${ }^{\mathrm{c}} \mathrm{CK}$ available between 10 and 14 months for 48 patients. Anti-HMGCR: anti-HMG-CoA-reductase; JIIM: juvenile idiopathic inflammatory myositis; CK: creatine kinase; CMAS: Childhood Myositis Assessment Score, 0-53; PGA: physician's global assessment visual analog score of disease activity, 0-10; IQR: interquartile range.

Personal non-commercial use only. The Journal of Rheumatology Copyright $\subset$ 2017. All rights reserved. 
dystrophy in the differential diagnosis, but electromyography and muscle biopsy were most consistent with an inflammatory myositis. While clinically this patient's myositis was not severe (CMAS 30-36, consistent with mild to moderate weakness and highest ever PGA 3), his CK has been persistently elevated. The patient had a poor response to MTX and ultimately responded clinically to RTX. CK remains $>1000$.

Patient 4. A 9.4-year-old girl presented with a 4-month history of progressive muscle weakness. An inflammatory rash was confined to her antecubital fossae. CMAS was 2, consistent with severe weakness. At presentation, alanine aminotransferase was $600 \mathrm{U} / 1$, lactate dehydrogenase $4000 \mathrm{U} / 1$, and CK 12,000. A liver biopsy was normal. MRI showed widespread muscle edema. Following treatment with IV methylprednisolone, PRED, and subcutaneous MTX, CMAS remained between 5 and 30 (moderate to severe weakness) and muscle enzymes were persistently elevated with CK $>3000$. Subsequent treatment included IFX, IV immunoglobulin (IG), mycophenolate mofetil (MMF), and
IV CYC. Her highest PGA was 9.5. The poor treatment response ultimately prompted diagnostic reassessment with muscle biopsy, skin biopsy, and weaning of all immunosuppression. The patient deteriorated without immunosuppression and PRED was reintroduced. Muscle biopsy showed necrosis and inflammation, and while not typical was felt to be consistent with myositis. Skin biopsy revealed inflammation in the dermis favoring DM. Following confirmation of anti-HMGCR status, the patient has been retreated with IVIG and MMF with a slow, but clear improvement.

Muscle biopsy. Muscle biopsy material was available for reassessment in 3 patients and the written report of the fourth biopsy was also available. Biopsy score components are shown in Table 2. The biopsies had similar and distinctive features with widespread variation in fiber size, regenerating fibers, necrotic fibers, and degenerating fibers. There were frequent fibers expressing immature isoforms of myosin and deposition of the membrane attack complex (MAC; C5b-C9) on the sarcolemma of many fibers. There were patchy dense

Table 2. Muscle biopsy score breakdown for patients with anti-HMGCR. Biopsy appearances were consistent with a destructive myopathy with high scores in the muscle fiber domain. All biopsies had evidence of inflammation. Muscle biopsies were analyzed using the JDM muscle biopsy score tool as previously described by Wedderburn, et $a l^{8,9}$.

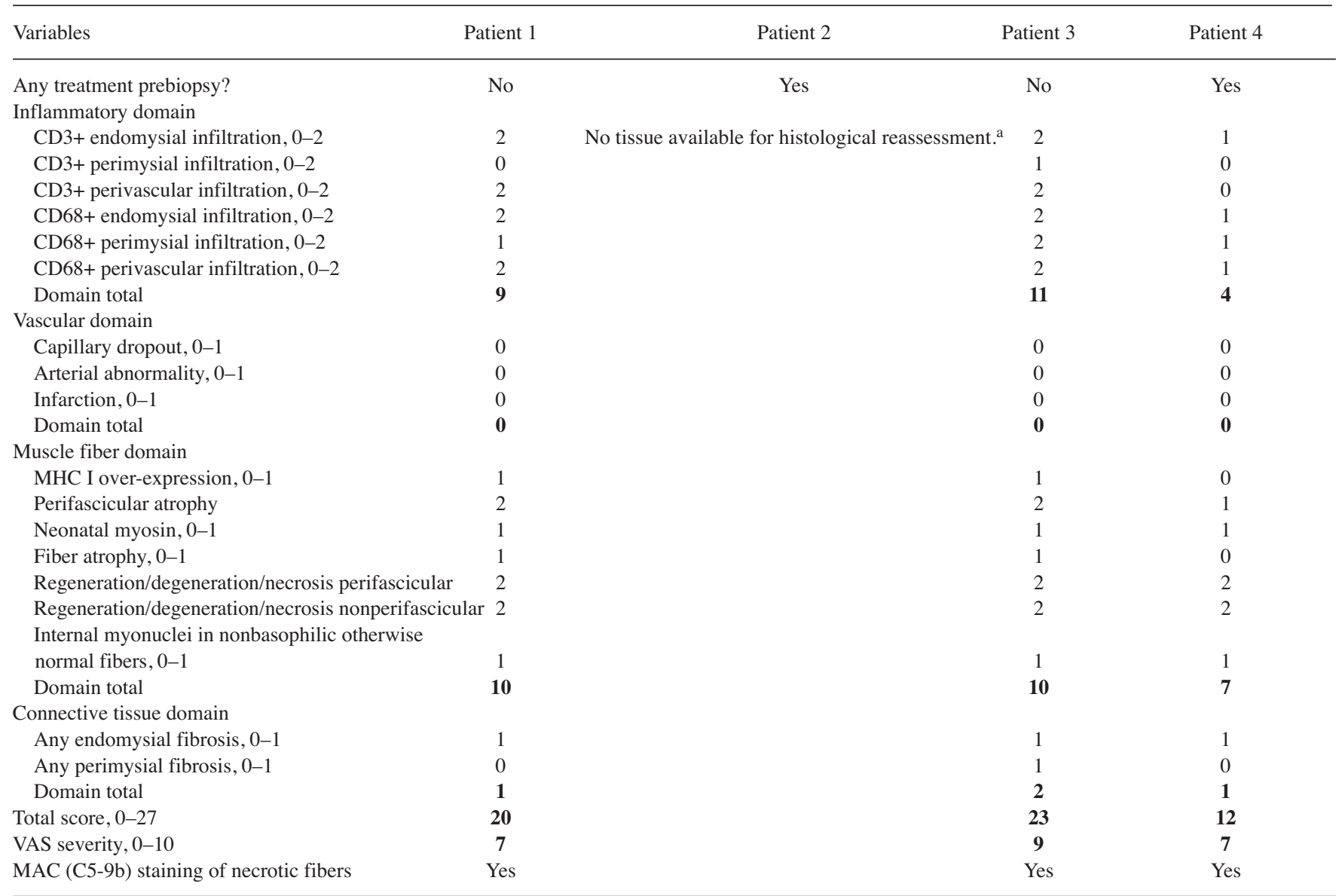

Significant data are in bold face. ${ }^{\text {a }}$ Original muscle biopsy report comments on muscle fiber necrosis and inflammation. Biopsy findings were considered supportive of a diagnosis of a myositis in the correct clinical context, but not diagnostic on their own. Anti-HMGCR: anti-HMG-CoA-reductase; JDM: juvenile dermatomyositis; VAS: visual analog scale; MAC: membrane attack complex.

Personal non-commercial use only. The Journal of Rheumatology Copyright @ 2017 . All rights reserved 
infiltrates of chronic inflammatory cells within the fascicles (Figure 1).

\section{DISCUSSION}

Consistent with previous reports, patients with anti-HMGCR presented with either profound or insidious onset muscle weakness ${ }^{3}$. Unlike affected adults, however, half of our patients had cutaneous disease $e^{2,3}$. Lack of skin involvement is unusual in JIIM and minimal/absent rash contributed to the need to actively exclude alternative diagnoses in those with anti-HMGCR. Remarkably, all children with anti-HMGCR underwent muscle biopsy and 2 electromyography; these invasive procedures are not routinely used diagnostically in the United Kingdom for suspected JIIM, the majority of patients being diagnosed on the basis of clinical features plus laboratory and MRI results.

Muscle biopsies were distinctive and features included those previously reported by Allenbach, et al, with muscle fiber necrosis and degeneration/regeneration ${ }^{3}$. Contrary to previous reports of a pauciimmune necrotizing myopathy, however, there was evidence of inflammation in all 4 biopsies. JIIM muscle biopsies can display a wide range of changes that may not necessarily be considered "typical" of DM. All patients with anti-HMGCR had an abnormal muscle biopsy with a pattern of histological features that, while not classic for DM, was consistent and distinctive. Recognition of this phenotype is important to ensure that muscle biopsy can provide the maximum level of diagnostic accuracy. Earlier recognition of anti-HMGCR autoantibodies may aid diagnosis, prevent potentially unnecessary investigations, and facilitate rapid treatment escalation in the absence of a good response to standard therapy. Mammen, et al confirmed the absence of anti-HMGCR in patients with genetic muscle diseases that often form the differential diagnosis ${ }^{13}$.

While rare, anti-HMGCR identify an important group of JIIM patients with severe disease necessitating aggressive treatment. In the United Kingdom, strict guidelines exist for funding biologic treatments, and it is remarkable that all 4 cases ultimately received this; in comparison with just $20.7 \%$ of the total cohort. We noted similarities in disease presentation to those patients with anti-SRP autoantibodies, in whom muscle weakness is also greater and cutaneous disease less common. Less than one-third of patients with anti-SRP, however, required treatment with a biologic drug and/or IV CYC. It is interesting to note that while statinexposed adult patients with anti-HMGCR are reported to respond dramatically to treatment, statin-naive patients have been noted to be younger, and may be refractory to immunosuppressive therapy ${ }^{2,14}$. Younger, statin-naive patients with anti-HMGCR may represent a separate subgroup of patients who are less responsive to treatment. These data suggest that in those children with anti-HMGCR and severe weakness, there is an argument for the early use of aggressive immunosuppression.

\section{ACKNOWLEDGMENT}

The Juvenile Dermatomyositis Research Group thanks all the patients and their families who contributed to the UK Juvenile Dermatomyositis Cohort and Biomarker Study.
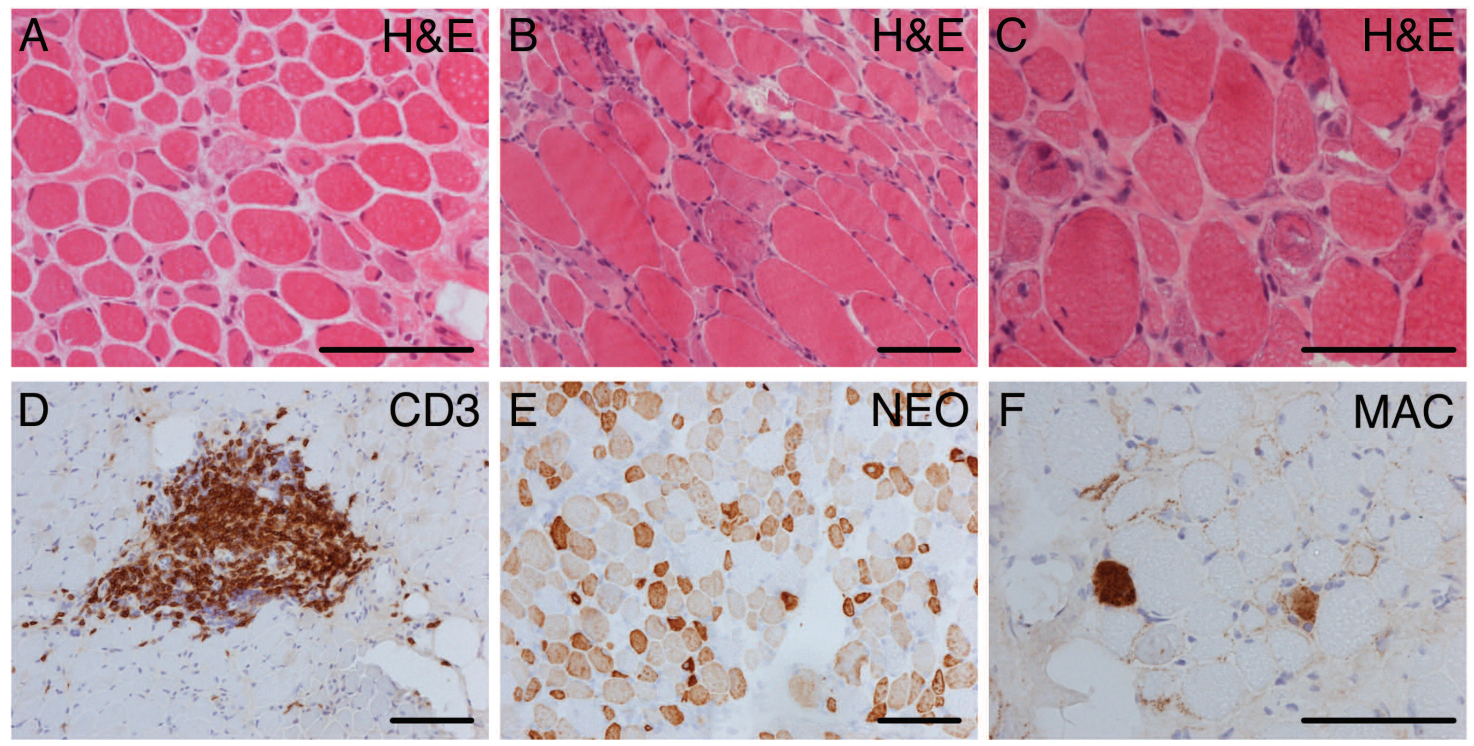

Figure 1. Muscle biopsy features of patients with anti-HMGCR autoantibodies. All 3 biopsies showed a similar distinctive pattern of pathology characterized by widespread variation in fiber size, regenerating fibers, necrotic fibers, and degenerating fibers with vacuoles and ill-defined eosinophilic granules (A-C). The abnormal fibers were present throughout all the fascicles with no perifascicular accentuation. There were patchy dense infiltrates of chronic inflammatory cells within the fascicles. The infiltrate showed a prominent component made up of T lymphocytes (D; CD3). There were fewer inflammatory cells in the perimysium. In all 3 cases, many of the fibers expressed a developmental isoform of myosin (E; NEO). Staining for the MAC (C5b-9) of the complement cascade highlighted necrotic fibers, but also outlined the sarcolemma of many of the non-necrotic fibers (F). Capillary deposition of MAC was not seen. Scale bars-100 $\mu \mathrm{m}$. Anti-HMGCR: anti-HMG-CoA-reductase; NEO: neonatal myosin; MAC: membrane attack complex.

Personal non-commercial use only. The Journal of Rheumatology Copyright @ 2017 . All rights reserved. 


\section{APPENDIX 1.}

List of study collaborators. Members of the Juvenile Dermatomyositis Research Group who contributed: Dr. Kate Armon, Joe Ellis-Gage, Holly Roper, Vanja Briggs, and Joanna Watts (Norfolk and Norwich University Hospitals); Dr. Liza McCann, Ian Roberts, Dr. Eileen Baildam, Louise Hanna, Olivia Lloyd, and Susan Wadeson (The Royal Liverpool Children's Hospital, Alder Hey, Liverpool); Dr. Phil Riley and Ann McGovern (Royal Manchester Children's Hospital, Manchester); Dr. Clive Ryder, Janis Scott, Beverley Thomas, Professor Taunton Southwood, and Dr. Eslam Al-Abadi (Birmingham Children's Hospital, Birmingham); Dr. Sue Wyatt, Gillian Jackson, Dr. Tania Amin, Dr. Mark Wood, Dr. Vanessa VanRooyen, and Deborah Burton (Leeds General Infirmary, Leeds); Dr. Joyce Davidson, Dr. Janet Gardner-Medwin, Dr. Neil Martin, Sue Ferguson, Liz Waxman, and Michael Browne (The Royal Hospital for Sick Children, Yorkhill, Glasgow); Dr. Mark Friswell, Professor Helen Foster, Alison Swift, Dr. Sharmila Jandial, Vicky Stevenson, Debbie Wade, Dr. Ethan Sen, Dr. Eve Smith, Lisa Qiao, Stuart Watson, and Claire Duong (Great North Children's Hospital, Newcastle); Dr. Helen Venning, Dr. Rangaraj Satyapal, Elizabeth Stretton, Mary Jordan, Dr. Ellen Mosley, Anna Frost, Lindsay Crate, Dr. Kishore Warrier, and Stefanie Stafford (Queens Medical Centre, Nottingham); Professor Lucy Wedderburn, Dr. Clarissa Pilkington, Dr. Nathan Hasson, Sue Maillard, Elizabeth Halkon, Virginia Brown, Audrey Juggins, Dr. Sally Smith, Sian Lunt, Elli Enayat, Hemlata Varsani, Laura Kassoumeri, Laura Beard, Katie Arnold, Yvonne Glackin, Stephanie Simou, Dr. Beverley Almeida, Dr. Kiran Nistala, Dr. Raquel Marques, Dr. Shireena Yasin, Dr. Claire Deakin, Stefanie Dowle, and Charis Papadopoulou (Great Ormond Street Hospital, London); Dr. Kevin Murray (Princess Margaret Hospital, Perth, Western Australia); Dr. John Ioannou and Linda Suffield (University College London Hospital, London); Dr. Muthana Al-Obaidi, Helen Lee, Sam Leach, Helen Smith, Dr. Anne-Marie McMahon, Heather Chisem, and Ruth Kingshott (Sheffield's Children's Hospital, Sheffield); Dr. Nick Wilkinson, Emma Inness, Eunice Kendall, David Mayers, Ruth Etherton, and Dr. Kathryn Bailey (Oxford University Hospitals, Oxford); Dr. Jacqui Clinch, Natalie Fineman, and Helen Pluess-Hall (Bristol Royal Hospital for Children, Bristol); Lindsay Vallance (Royal Aberdeen Children's Hospital); Louise Akeroyd (Bradford Teaching Hospitals); Dr. Alice Leahy, Amy Collier, Rebecca Cutts, Dr. Hans De Graaf, Dr. Brian Davidson, Sarah Hartfree, and Danny Pratt (University Hospital Southampton).

\section{REFERENCES}

1. Liang C, Needham M. Necrotizing autoimmune myopathy. Curr Opin Rheumatol 2011;23:612-9.

2. Mammen AL, Chung T, Christopher-Stine L, Rosen P, Rosen A, Doering KR, et al. Autoantibodies against 3-hydroxy-3-methylglutaryl-coenzyme A reductase in patients with statin-associated autoimmune myopathy. Arthritis Rheum 2011;63:713-21.

3. Allenbach Y, Drouot L, Rigolet A, Charuel JL, Jouen F, Romero $\mathrm{NB}$, et al. Anti-HMGCR autoantibodies in European patients with autoimmune necrotizing myopathies: inconstant exposure to statin. Medicine 2014;93:150-7.
4. Martin N, Krol P, Smith S, Murray K, Pilkington CA, Davidson JE, et al; Juvenile Dermatomyositis Research Group. A national registry for juvenile dermatomyositis and other paediatric idiopathic inflammatory myopathies: 10 years' experience; the Juvenile Dermatomyositis National (UK and Ireland) Cohort Biomarker Study and Repository for Idiopathic Inflammatory Myopathies. Rheumatology 2011;50:137-45.

5. Tansley SL, Betteridge ZE, Shaddick G, Gunawardena H, Arnold K, Wedderburn LR, et al. Calcinosis in juvenile dermatomyositis is influenced by both anti-NXP2 autoantibody status and age at disease onset. Rheumatology 2014;53:2204-8.

6. Tansley SL, Betteridge ZE, Gunawardena H, Jacques TS, Owens CM, Pilkington C, et al; UK Juvenile Dermatomyositis Research Group. Anti-MDA5 autoantibodies in juvenile dermatomyositis identify a distinct clinical phenotype: a prospective cohort study. Arthritis Res Ther 2014;16:R138.

7. Klein M, Mann H, Pleštilová L, Zámečník J, Betteridge Z, McHugh $\mathrm{N}$, et al. Increasing incidence of immune-mediated necrotizing myopathy: single-centre experience. Rheumatology 2015; 54:2010-4.

8. Varsani H, Charman SC, Li CK, Marie SK, Amato AA, Banwell B, et al; UK Juvenile Dermatomyositis Research Group. Validation of a score tool for measurement of histological severity in juvenile dermatomyositis and association with clinical severity of disease. Ann Rheum Dis 2015;74:204-10.

9. Wedderburn LR, Varsani H, Li CK, Newton KR, Amato AA, Banwell B, et al; UK Juvenile Dermatomyositis Research Group. International consensus on a proposed score system for muscle biopsy evaluation in patients with juvenile dermatomyositis: a tool for potential use in clinical trials. Arthritis Rheum 2007; 57:1192-201.

10. McCann LJ, Juggins AD, Maillard SM, Wedderburn LR, Davidson JE, Murray KJ, et al; Juvenile Dermatomyositis Research Group. The Juvenile Dermatomyositis National Registry and Repository (UK and Ireland) - clinical characteristics of children recruited within the first 5 yr. Rheumatology 2006;45:1255-60.

11. Pachman LM, Hayford JR, Chung A, Daugherty CA, Pallansch MA, Fink CW, et al. Juvenile dermatomyositis at diagnosis: clinical characteristics of 79 children. J Rheumatol 1998;25:1198-204.

12. Huber AM, Feldman BM, Rennebohm RM, Hicks JE, Lindsley CB, Perez MD, et al; Juvenile Dermatomyositis Disease Activity Collaborative Study Group. Validation and clinical significance of the Childhood Myositis Assessment Scale for assessment of muscle function in the juvenile idiopathic inflammatory myopathies. Arthritis Rheum 2004;50:1595-603.

13. Mammen AL, Casciola-Rosen L, Christopher-Stine L, Lloyd TE, Wagner KR. Myositis-specific autoantibodies are specific for myositis compared to genetic muscle disease. Neurol Neuroimmunol Neuroinflamm 2015;2:e172.

14. Mammen AL. Necrotizing myopathies: beyond statins. Curr Opin Rheumatol 2014;26:679-83. 


\section{Correction}

Anti-HMGCR Autoantibodies in Juvenile Idiopathic Inflammatory Myopathies Identify a Rare but Clinically Important Subset of Patients

Tansley SL, Betteridge ZE, Simou S, Jacques TS, Pilkington C, Wood M, et al. Anti-HMGCR autoantibodies in juvenile idiopathic inflammatory myopathies identify a rare but clinically important subset of patients. J Rheumatol 2017; doi:10.3899/jrheum.160871. In the online version of this article, the name of the lead author and the collaborating study group were omitted from the byline. The lead author is Neil J. McHugh, on behalf of the Juvenile Dermatomyositis Research Group.

This correction has been made to the online version of the article. It should be noted that the print version of the article, and the PDF version (available online), are correct.

doi:10.3899/jrheum.160871.C1 